Revista de Psicología Vol. 39 (2), 2021 (e-ISSN 2223-3733)

\title{
Inmersión y activación de estados emocionales con videojuegos de realidad virtual
}

\author{
David Torres Fernández ${ }^{1}$, Emanuel Blanca Moya², Rolando Pérez Sánchez ${ }^{3}$ \\ Universidad de Costa Rica-Costa Rica
}

El estudio se dirigió a estudiar la asociación entre inmersión en videojuegos de Realidad Virtual (RV) y la activación emocional. Se llevó a cabo un diseño experimental intrasujetos, con 97 estudiantes universitarios. Se llevaron a cabo mediciones de frecuencia cardiaca y del estado emocional percibido. Los resultados mostraron que la condición RV presenta mayor inmersión y mayor frecuencia cardiaca que la condición no RV. Los estados emocionales percibidos fueron predictores de la inmersión en ambas condiciones, teniendo que en la condición no RV, mayores puntajes en valoración de las emociones positivas y negativas se asociaron a una mayor inmersión, mientras que en RV solo las emociones positivas se relacionaron con la inmersión. Se discute la relevancia de estos resultados.

Palabras clave: Inmersión, activación emocional, videojuegos, realidad virtual

\section{Immersion and Emotional Arousal with Virtual Reality Videogames}

This research studies the association between immersion in Virtual Reality (VR) videogames and emotional activation. Responses to a videogame in a VR setting and in a no VR setting were compared. The study uses a within-subject design with 97 university students. Heart rate and self-perceived emotional state were measured. Results show higher immersion levels and heart rate in the VR condition. Perceived emotional state predicted immersion in both conditions, nevertheless, in the no VR condition higher scores in both, positive and negative, emotions were related with higher immersion experience, while in the VR condition only positive emotions were related with higher immersion. The implications and relevance of these findings are discussed.

Keywords: Immersion, emotional activation, videogames, virtual reality.

1 Licenciado en Psicología. Profesor-investigador de la Universidad de Costa Rica, Costa Rica. Dirección postal: Instituto de Investigaciones Psicológicas, Universidad de Costa Rica. Apartado postal: 11501-2060. Contacto: datofez1@yahoo.com https://orcid.org/0000-0003-3023-5129

2 Licenciado en Psicología. Investigador de la Universidad de Costa Rica, Costa Rica. Dirección postal: Instituto de Investigaciones Psicológicas, Universidad de Costa Rica. Apartado postal: 11501-2060. Contacto: emanuel.blanca@gmail.com https:/orcid.org/0000-0001-8194-7797

3 Doctor en Sociología. Profesor catedrático en la Universidad de Costa Rica, Costa Rica. Dirección postal: Instituto de Investigaciones Psicológicas, Universidad de Costa Rica. Apartado postal: 11501-2060. Contacto: rolarez@gmail.com https://orcid.org/0000-0001-6321-2543 


\section{Imersão e Ativação de Estados Emocionais com Videojogo de Realidade Virtual}

$\mathrm{O}$ estudo objetivou estudar a associação entre a imersão em videojogo de Realidade Virtual (RV) e a ativação emocional. Mediante um delineamento experimental de medidas repetidas com 97 estudantes universitários, em que se aplicaram medidas de frequência cardíaca e estado emocional percibido. Os resultados mostraram que os estudantes universitários apresentaram maior imersão e maior frequência cardíaca na condição RV que na sem RV. Os estados emocionais percebidos foram preditores da imersão em ambas condiçóes de jogo, tendo que para a condição sem RV, pontuaçóes mais elevadas tanto para ativação de emoçóes positivas quanto negativas se associaram a uma maior imersão, enquanto na condição RV somente as emoçóes positivas se relacionaron com a imersão. A relevância dos resultados é discutida.

Palavras-chave: Imersão, ativação emocional, videojogo, realidade virtual

\section{Immersion et activation des états émotionnels avec les jeux vidéo de réalité virtuelle}

Cette étude avait comme objectif d'étudier l'association entre l'immersion dans les jeux vidéo en Réalité Virtuelle (RV) et l'activation emotionnelle. 97 étudiants universitaires ont participé à cette étude. Sur la base d'un design intra-sujets, il a été mesuré la fréquence cardiaque et états émotionnels perçus. Les résultats ont montré une immersion plus intense et une la fréquence cardiaque plus élevée dans la condition de RV comparée à la sans-RV. Les états émotionnels perçus ont servi de prédicteurs de l'immersion dans les deux jeux. Cependant, pour sans-VR, des scores plus élevés en émotions positives et négatives ont été associés à une immersion plus grande; alors que pour VR, seules les émotions positives ont été associés à l'immersion. La pertinence de ces résultats est discutée.

Mots-clés: Immersion, activation émotionnelle, jeux vidéo, réalité virtuelle 
En los últimos años, la investigación sobre los usos y efectos de los videojuegos ha aumentado considerablemente (Griffiths, Király, Pontes y Demetrovics, 2015; Nuyens, Kuss, Lopez-Fernandez y Griffiyhs, 2018; Griffiths Halbrook, O’Donnell, y Msetfi (2019). Utilizando diferentes metodologías, se han estudiado sus aportes a diferentes variables psicológicas y su relación con aspectos cognoscitivos y emocionales, en el autoconcepto, así como sus consecuencias sociales. Sin embargo, con la rápida evolución de su tecnología, su multidimensionalidad y las diferentes modalidades de juego, que se han convertido en una parte casi integral de la vida cotidiana, la definición misma de videojuegos sigue en proceso.

Una de las áreas de estudio más recientes en los videojuegos es el proceso de inmersión, también conocido como presencia espacial, el cual es un tema que presenta especial relevancia con el avance de la tecnología, aunque también ha sido estudiado en medios tradicionales como la televisión, el cine y la literatura (Lee, 2004). Lee (2004. p. 37) le define como "un estado psicológico en el que objetos virtuales son experimentados como objetos reales ya sea en formas sensoriales o no sensoriales" (Hoffer, Wirth, Kuehne, Schramm y Sacau, 2012; Wirth et al., 2015; Wirth, Hofer y Schramm, 2012).

La inmersión ha sido descrita como un modelo de dos pasos que, en un primer momento, involucra la construcción de un modelo mental del ambiente mediado, predicho por la atención y los rasgos individuales de la imaginación visual-espacial. Una vez creado este modelo mental surge la inmersión, la cual es mediada por el involucramiento cognitivo, la suspensión de la no creencia y el interés de dominio específico. Finalmente, esta inmersión está conformada por la auto-localización en la presencia espacial y las posibles acciones a seguir en dicha presencia. Cabe recalcar que los diferentes componentes de cada paso están a su vez compuesto por factores del usuario y del medio (Hoffer, Wirth, Kuehne, Schramm y Sacau, 2012; Wirth et al., 2015). 
En esta línea, controlando los factores antes predichos, se ha encontrado que la inmersión puede ser también predicha por el involucramiento emocional en ambientes virtuales y la absorción de rasgos (disposición a tener episodios de atención que compromete totalmente a la persona con sus recursos representacionales), de tal forma que mayores niveles de involucramiento emocional y de absorción de rasgos generan una mayor presencia espacial (Wirth, Hofer y Schramm, 2012). Además, la inmersión también se relaciona con mayores niveles de autonomía y competencia percibidas a la hora de jugar videojuegos; variables que a su vez se relacionan con su propio disfrute y con cambios en el bienestar subjetivo después de jugar (Ryan, Rigby y Przybylski, 2006). Esto es afirmado por otros estudios donde el disfrute de los videojuegos se puede predecir poderosamente por la inmersión que el juego puede generar, tanto al jugar en consolas como en móviles (Shafer y Carbonara, 2015).

Uno de los aspectos más novedosos en los videojuegos de consola es la incorporación de la realidad virtual (RV o VR por sus siglas en inglés), la cual ha sido utilizada desde hace algunos años en ambientes digitales, principalmente en computadoras y más recientemente en teléfonos móviles, y ha sido investigada como una herramienta para la rehabilitación de las funciones de las extremidades superiores y el balance en personas que han sufrido de derrames cerebrales (de Rooij, van de Port y Meijer, 2016; Ji y Lee, 2016; Yamato, Pompeu, Pompeu y Hasset, 2016). Sin embargo, en estos casos la realidad virtual se ha utilizado con programas diseñados para la terapia y no con videojuegos propiamente.

Tomando en cuenta la relación entre activación emocional, involucramiento e inmersión, se podría asumir el papel de la inmersión en la RV a la hora de utilizar mediciones fisiológicas para evaluar la respuesta de los usuarios ante los eventos que se le presenten. Así, con la tecnología RV y midiendo la variación en la frecuencia cardiaca, se ha encontrado que los procesos de inmersión en la RV generan una mayor actividad cardíaca que el observar películas en tercera dimensión o 3D (Malinska, Zuzewicz, Bugajska, \& Grabowski, 2015). De igual 
forma, la medición de estas variaciones ha sido utilizada en estudios terapéuticos para el tratamiento del Síndrome de Estrés Postraumático, encontrando un aumento en el ritmo cardíaco en situaciones de miedo generadas en ambiente virtuales de guerra (Roy et al., 2013) En esta línea la RV se ha utilizado evaluando los niveles de ansiedad, a través de la activación fisiológica, en diferentes estudios, por ejemplo para evaluar los niveles de ansiedad en situaciones sociales desarrolladas en ambientes sociales simulados en realidad virtual (Hartanto et al., 2014); para evaluar los niveles de ansiedad ante la presencia de arañas en un grupo de sujetos con aracnofobia (Notzon et al., 2015); para medir el papel de la activación fisiológica en las terapias de exposición con medios virtuales, exponiendo sujetos con acrofobia un grupo control a una simulación de altura, en la cual se obtuvo activación fisiológica en ambos grupos (Diemer, Lohkamp, Mühlberger, \& Zwanzger, 2016). Además de medir la activación fisiológica para evaluar conductas de riesgos en adolescentes, simulando situaciones de abuso de sustancias, riesgos sexuales en ambientes de fiesta virtuales (Hadley et al., 2014).

Las investigaciones en realidad virtual, como se ha mencionado antes, se han realizado en ambientes controlados y específicos a ambientes clínicos, dejando de lado los efectos que pueda tener en los usuarios de estas tecnologías al utilizarlo de manera más cotidiana en sus hogares, además de la ausencia de evaluación del impacto emocional de los videojuegos en modalidad virtual. Por esta razón, es que se torna importante el evaluar el papel de los juegos de realidad virtual en la inmersión y activación emocional en los videojuegos. Al respecto se identificó solo un estudio de Roettl y Terlutter (2018) que encuentran mayor experiencia de presencia y mayor carga cognitiva en juegos de Realidad Virtual en comparación con juegos 3D o 2D.

En esta investigación se pretende dar contenido a los vacíos investigativos hallados, al comparar la activación emocional en videojuegos comerciales en modalidad virtual con la no virtual, así como al estudiar el uso de videojuegos en un ambiente más cotidiano, similar al que se experimenta al jugar en el hogar. Así, la presente investigación se enfoca en el estudio de la inmersión en un videojuego de realidad virtual y el 
cómo puede influir esta en la activación emocional, comparándolo con el uso de videojuegos sin la modalidad de realidad virtual.

Las hipótesis del presente estudio serían las siguientes: H1: Los videojuegos con la modalidad de realidad virtual generan mayor inmersión que los videojuegos sin modalidad de realidad virtual. H2: Los videojuegos con la modalidad de realidad virtual generan mayor activación emocional que los videojuegos sin modalidad de realidad virtual. H3: La valoración de los estados emocionales percibidos se van a asociar con la Inmersión en ambas condiciones de juego. H4: Los videojuegos con la modalidad de realidad virtual generan mayor activación fisiológica que los videojuegos sin modalidad de realidad virtual.

El género se incorporó como una variable control al considerarlo relevante para la investigación, pues aunque no es el tema central de esta, son relevantes de determinar ya que, según Lopez-Fernandez, Williams, Griffiths, \& Kuss (2019), contribuye a identificar las diferencias entre hombres y mujeres en la temática de los video juegos, así como los factores socio-culturales asociados.

\section{Método}

\section{Participantes}

Para este estudio se trabajó con estudiantes universitarios, logrando una participación de 97 estudiantes universitarios (70\% hombres), con edades entre los 18 y 36 años $(M=22.19, \mathrm{DE}=3.55)$. Se tenía como como criterio principal de inclusión el contar con experiencia previa en el uso de consolas de videojuegos, aún cuando no juegue actualmente con la misma, principalmente PlayStation 4. En ese sentido, respecto al dominio de la consola, aunque todos tienen una experiencia previa con la consola, un $41 \%$ asegura tener un dominio regular, un $39 \%$ dominio total y un $20 \%$ escaso dominio de la consola. Con respecto a la frecuencia de uso de videojuegos, se reporta un uso semanal entre 0 
y 75 horas $(M=19.80, D E=15.06)$. La participación en dicho estudio fue voluntaria.

Con el fin de evitar riesgos en la salud de los participantes y basándose en estudios anteriores (Guerrero y Valero, 2013; Ortiz de Gortari y Griffiths, 2015), se excluyeron del estudio a personas que padecían de fotosensibilidad, migrañas alucinatorias, trastornos de ansiedad, enfermedades cardíacas, esquizofrenia, epilepsia, mareos o nauseas.

Todas las personas participantes firmaron un consentimiento informado, en donde se les explicó el procedimiento, la presencia de posibles riesgos y las medidas para afrontarlos. El estudio fue aprobado por el Comité Ético Científico de la Universidad de Costa Rica, al cual está adscrito este proyecto de investigación.

\section{Instrumentos}

Con el fin de evaluar la activación emocional se recurrieron a dos medidas:

Estados de ánimo percibidos: se recurrió a la "Lista de afectos positivos y negativos" (PANAS) desarrollada por Watson, Clark, y Tellegen (1988), en la versión al español y adaptación a Costa Rica de Gutierrez-Doña (2003). Este instrumento consta de dos subescalas, afecto positivo y afecto negativo, las cuales constan de una lista de adjetivos que deben de calificarse en una escala de 4 puntos según la presencia de cada estado de ánimo (de mucho a nada). Esta se aplicaba al inicio y final del experimento. Las medidas de los alfas de Cronbach mostraron valores entre .808 y .870 (puntuación de 1 a 4) en el caso del PANAS positivo, y valores entre .841 y .860 (puntuación de 1 a 4), en el caso del PANAS negativo.

Frecuencia cardiaca: se recurrió al registro de la frecuencia cardiaca para medir la activación fisiológica asociada a las condiciones experimentales. Para medir la frecuencia cardiaca durante el juego se recurrió a un dispositivo de monitoreo usado en la muńeca de la mano, el cual estaba sincronizado a una aplicación de teléfono celular. La frecuencia cardiaca (activación fisiológica) se calculó a partir del promedio de 
las frecuencias cardiacas registradas durante el juego, medidas a cada minuto de juego, iniciando por un primer registro inmediatamente antes de jugar.

Así también se tomaron en cuenta otros factores de control, valorados con instrumentos específicos para ellos:

Intensidad de uso de video-juegos: consta de un auto-reporte sobre horas de juego por semana.

Experiencia de uso de los juegos en estudio: auto-reporte sobre nivel de conocimiento de videojuegos en una escala de 0 a 10.

Escala sobre Inmersión en los videojuegos: Se utilizó una versión adaptada del Game Immersion Questionnaire (Cheng, Shet y Anneta, 2014), el cual fue traducido al español y evaluado a través de entrevista cognitiva con el fin de ponerlo a prueba y ofrecer también una versión validada como producto de este proyecto de investigación. El cuestionario original cuenta con 24 ítems que mide los tres niveles de inmersión (compromiso, engrosamiento y inmersión total) y se responden en un formato Likert de 5 puntos. En la versión adaptada, se cuenta con la misma cantidad de ítems pero adecuados en términos de idioma y de interpretación asegurando su comprensión en contexto. Esta escala se aplicaba al inicio y final del experimento, tanto en condición de RV como de No RV. Aquí los alfa de Cronbach mostraron valores entre .85 y .86 (puntuación de 1 a 5).

Selección del videojuego y equipo utilizado

Para el desarrollo de esta investigación se recurrió al uso de una consola Play Station 4, con un control remoto, un dispositivo Play Station RV (compuesto por una cámara específica para esta consola, audífonos y un casco especial para generar la experiencia de realidad virtual) y un videojuego que tenía la posibilidad de jugarse tanto con modalidad RV, como sin modalidad RV, de manera que se pudiera asegurar la comparabilidad exacta entre ambas experiencias, acorde a los objetivos de la investigación.

Para lograr la selección final del juego, además de cerciorarse de que cumpliera con el criterio anterior, se realizó una exploración exhaustiva de múltiples tipos y géneros (acción, puzles o acertijos, de 
peleas, carreras de autos, shooters o de disparar, etc.). Esto llevó a una primera propuesta de videojuegos que luego fueron valorados considerando los siguientes criterios: capacidad de inmersión (entendido como "la capacidad que tiene el videojuego de involucrar al jugador en un ambiente tan envolvente dentro del juego que pueda sentirse como parte del mismo"), nivel de entretenimiento ofrecido o "fun factor" (qué tan entretenido o no es el juego específicamente durante los minutos y "pantalla" disponibles), dificultad (qué tan difícil se considera el juego según las características de la pantalla jugada, la misión por atender o reto por resolver o vencer), conocimiento previo requerido (qué tan necesario es conocer o haber jugado algún juego similar, en términos de captar con facilidad su lógica, el manejo de su control y la comprensión de cómo proceder para avanzar), la jugabilidad (facilidad y naturalidad que ofrece el juego para el manejo de la cámara, el uso del control y el movimiento del personaje) y el nivel de riesgos potenciales (cualquier tipo de aversión potencial que presenta el juego y que pueda resultar en riesgos para la salud física o emocional, tales como niveles de violencia, generación natural de vértigo o mareos o elementos ofensivos de cualqueir índole).

Para validar esto, se contó con un jueceo experto por parte de un grupo de investigadores en Psicología con experiencia en la temática, quienes para cada juego ofrecido revisaron el cumplimiento de dichos criterios utilizando una escala de valoración 0 a 10 (siendo esta la puntuación que reflejaría un cumplimiento máximo). En el caso específico del nivel de riesgos potenciales, contrario a los demás criterios, se esperaba una puntuación de 0 . Esto llevó a la selección preliminar de dos juegos.

\section{Procedimiento}

Se realizaron pruebas piloto comparando los dos juegos antes seleccionados (ambos ofreciendo la opción de RV/No RV), y aplicando los demás instrumentos de investigación ya descritos, para luego validarlos con una entrevista cognitiva que permitió revisar cada escala y 
cuestionario aplicado. De esta manera se logró corroborar la comprensión y claridad de las instrucciones y planteamiento de cada ítem del instrumental. Este pilotaje se llevó acabo con participantes que eran tanto jugadores casuales como expertos (activos en el gremio de los videojuegos, con muchas horas de juego y con participación en eventos internacionales). Tras esta prueba piloto, se obtuvo la versión final de los instrumentos de medición y se seleccionó el video juego Resident Evil VII para el experimento, debido al alto cumplimiento con los criterios antes descritos y los niveles de inmersión que propone.

Con este videojuego y las versiones finales de los instrumentos, se realizó un estudio experimental de mediciones repetidas en el cual los participantes jugaron en las dos condiciones: en modalidad de realidad virtual (RV) y sin la modalidad de realidad virtual (No RV). Se aleatorizó el orden de inicio de las condiciones para cada participante, así como las pantallas que jugaría. Para esto se contaba con dos pantallas previamente seleccionadas con las que podría jugar. El experimento se realizó en una sala acondicionada para dicho fin en las instalaciones del Instituto de Investigaciones Psicológicas, de la Universidad de Costa Rica.

El procedimiento iniciaba con el llenado del cuestionario sobre estados de ánimo (PANAS); el instrumento de intensidad de uso de video-juegos y el reporte sobre experiencia de uso de los juegos en estudio. Seguidamente se le colocaba al participante el sistema de medición de su ritmo cardíaco, el cual utilizará hasta el final del experimento. Asimismo, se colocaba el equipo de RV, en el caso en que fuera esa la condición con la que iniciaría, y posteriormente se daba un periodo de entrenamiento en donde se le explica el juego y se le dan 5 minutos de juego en una pantalla abierta para familiarizarse con la tarea.

Una vez concluida esta fase a cada participante se le otorgaban 20 minutos de juego (o hasta que perdiera la vida o pasara la pantalla, lo que pasara primero) en una sección pre-elegida del videojuego Resident Evil VII. Esto era tiempo suficiente para ejecutar las diferentes posibilidades de movimiento que contempla un juego de acción. Seguidamente la persona llenaba nuevamente el cuestionario sobre estados 
de ánimo y la escala sobre inmersión de los videojuegos. Tanto en la fase de prueba como en el experimento propiamente (en ambas condiciones) se llevó el registro de frecuencia cardiaca por intervalos de un minuto. Cabe señalar para las condiciones se llevaron a cabo den dos días diferentes con una distancia de una semana entre una y otra; es decir, quien jugaba en condición RV en su primera experimentación, jugaría en modalidad No RV una semana después y viceversa.

\section{Análisis de datos}

Para analizar los resultados se utilizó el paquetes estadístico JASP y se realizaron análisis descriptivos, pruebas $T$ para muestras relacionadas, correlaciones bivariadas y regresiones lineales múltiples. Al ser un estudio experimental, y tomando en cuenta que los principales análisis realizados están baasados en el estadístico F, la normalidad se considera irrelevante ya que el estadístico $\mathrm{F}$ es lo suficientemente robusto en términos de error Tipo I en casos de no normalidad (Blanca et al., 2017).

\section{Resultados}

Se realizaron anovas de medidas repetidas para determinar los cambios en la activación emocional percibida y su relación con el género del participante, tanto para la condición RV como No RV.

Como parte de la evaluación de la activación emocional se tomaron en cuenta las diferencias en los puntajes en emociones positivas y negativas antes y después de la experiencia de juego. En el caso de la activación emocional positiva, en la condición de RV, se encontraron diferencias significativas $\left(F_{1,95}=5.467, p=.021, \eta^{2}=.054\right.$, IC $5 \%$ $[-.200,-.022])$, donde la condición RV presenta un mayor puntaje en la activación emocional positiva después de jugar $(M=2.83, D T=.53)$ en comparación con el antes de jugar $(M=2.72, D T=.51)$. Sin embargo, no se observaron diferencias por género. En la condición No RV no se encontraron diferencias significativas. 
En cuanto a la activación emocional negativa, en la condición No RV se encontraron diferencias significativas $\left(F_{1,95}=25.255, \mathrm{p}<.001\right.$, $\eta^{2}=.210$, IC 95\% [-.344, -.147]), donde hay un aumento en la activación emocional negativa después de jugar $(\mathrm{M}=1.63$, DT=.52) en comparación a antes de jugar $(M=1.38, D T=.42)$. En este caso también se observaron diferencias por género $\left(F_{1,95}=14.274, p<.001, \eta^{2}=.131\right.$, IC $95 \%[.152, .488])$, donde las mujeres puntúan significativamente más alto que los hombres.

Por último, en el caso de la activación emocional negativa en la condición RV también se encontraron diferencias significativas $\left(F_{1,95}=42.406, \mathrm{p}<.001, \eta^{2}=.309\right.$, IC $\left.95 \%[-.432,-.220]\right)$, donde después de jugar se registra mayor activación emocional negativa $(M=$ $1.74, D T=.52)$ que antes de jugar $(M=1.41, D T=.40)$. De igual forma se encontraron diferencias en la interacción género-tiempo de medición $\left(F_{1,95}=4.169, p=.044, \eta^{2}=.042\right)$ con puntajes más altos después de jugar, tanto de hombres como mujeres, con relación al antes de jugar en ambos géneros. No se encontraron diferencias significativas por género.

Para determinar la presencia de diferencias en la inmersión entre las condiciones RV y No RV se recurrió a pruebas T para muestras relacionadas. Los análisis muestran diferencias significativas $\left(T_{96}=-\right.$ $8.08, p<.001, d=-.65$, IC $(95 \%)[-.84,-.51]$, con mayores puntajes de inmersión en la condición RV $(M=3.78, D E=.60)$ que en la No RV $(M=3.40, D E=64)$.

Se recurrió al mismo procedimiento para determinar las diferencias en la Frecuencia Cardiaca entre ambas condiciones. Los resultados muestran diferencias significativas $\left(T_{96}=2.07, p<.05, d=.21, I C 95 \%\right.$ $[.14, .69])$, donde se observan frecuencias cardiacas más altas en la condición $R V(M=44.11, D E=13.70)$ en comparación a la $N o R V$ $(M=40.60, D E=11.39)$. 


\section{Inmersión en Condición No RV}

Se realizó un análisis de correlaciones para determinar las variables que se asocian con la inmersión en esta condición (Tabla 1). El resultado muestra correlaciones positivas con la frecuencia de uso de PlayStation $(r=.20, p=.047)$, las emociones positivas después de jugar $(r=.33$, $p=.001)$ y las emociones negativas después de jugar $(r=.26, p=.010)$. No se encontraron relaciones significativas con las demás variables.

\section{Tabla 1}

Correlaciones para las variables en estudio en condición sin realidad virtual

\begin{tabular}{lcccccccc}
\hline & 1 & 2 & 3 & 4 & 5 & 6 & 7 & 8 \\
\hline 1. Inmersión & - & & & & & & \\
2. Activación fisiológica (FC) & -.03 & - & & & & & \\
3. Frecuencia de uso & $.20^{*}$ & $.28^{* *}$ & - & & & & \\
4. Edad & -.02 & $-.20^{*}$ & $-.24^{*}$ & - & & & \\
5. Sexo & -.02 & -.15 & .03 & $.20^{*}$ & - & & \\
6. Tiempo & .10 & .19 & .09 & .13 & -.03 & - & & \\
7. Emociones positivas & $.33^{* *}$ & -.01 & .19 & .06 & .11 & $.29^{* *}$ & - & \\
8. Emociones negativas & $.26^{* *}$ & $.25^{*}$ & .02 & -.05 & $-.34^{* *}$ & -.12 & -.07 & - \\
\hline
\end{tabular}

Nota. ${ }^{*} p<.05,{ }^{* *} p<.01,{ }^{* * *} p<.001$; Sexo: 1: Mujer, 2: Hombre

Seguido a esto se realizó un análisis de regresión lineal, utilizando el método stepwise, para determinar los predictores de la inmersión en esta condición (Tabla 2). El modelo final de la regresión explica un $17 \%$ de la varianza $\left(F_{2,93}=9.59, \mathrm{p}<.001, f=.20\right.$, IC 95\%[.05, .42] $)$ y muestra como principal variable predictora las emociones positivas después de jugar $(\beta=.38, p<.001)$, seguida de las emociones negativas $(\beta=.35, p=.003)$. 


\section{Tabla 2}

Predictores de la inmersión en condición sin realidad virtual

\begin{tabular}{lcccc}
\hline \multirow{2}{*}{ Variable } & \multicolumn{2}{c}{ Inmersión en condición sin realidad virtual } \\
\cline { 2 - 3 } \cline { 4 - 5 } \cline { 4 - 5 } & \multicolumn{2}{c}{ Modelo 1} & & \multicolumn{2}{c}{ Modelo 2} \\
\cline { 2 - 4 } Constante & 2.43 & & 1.82 & {$[1.131-2.508]$} \\
Emociones positivas & .36 & & .38 & {$[.176-.575]$} \\
Emociones negativas & & & .35 & {$[.117-.575]$} \\
$R_{2}$ & .11 & & & .17 \\
$F$ & $11.70^{* *}$ & & & $10.85^{*}$ \\
$\Delta R_{2}$ & & & & .078 \\
$\Delta F$ & & & & 9.001 \\
\hline
\end{tabular}

Nota. $\mathrm{N}=97, \mathrm{IC}=$ Intervalo de confianza. ${ }^{*} p<.01{ }^{* *} p<.001$. Se presentan solo las relaciones significativas.

\section{Inmersión en condición RV}

Se realizó un análisis de correlaciones para determinar las variables que se correlacionan con la inmersión en esta condición (Tabla 3). El resultado muestra una correlación positiva con las emociones positivas después de jugar $(r=.31, p=.002)$. No se encontraron relaciones significativas con las demás variables.

Seguido a esto se realizó un análisis de regresión lineal, utilizando el método stepwise, para determinar los predictores de la inmersión en esta condición (Tabla 4). El modelo final de la regresión explica un 8\% de la varianza $\left(F_{1,95}=9.95, \mathrm{p}=.002, f=.10\right.$, IC $\left.95 \%[-.01, .23]\right)$ y muestra como una variable predictora las emociones positivas después de jugar $(\beta=.34, \mathrm{p}<.002)$. 
Inmersión y activación de estados emocionales con videojuegos de realidad virtual / Torres et al.

\section{Tabla 3}

Correlaciones para las variables en estudio en condición con realidad virtual

\begin{tabular}{lcccccccc}
\hline & 1 & 2 & 3 & 4 & 5 & 6 & 7 & 8 \\
\hline 1. Inmersión & - & & & & & & & \\
2. Activación fisiológica (FC) & -.14 & - & & & & & \\
3. Frecuencia de uso & .08 & $.20^{*}$ & - & & & & \\
4. Edad & .11 & -.19 & -.17 & - & & & \\
5. Sexo & .05 & -.09 & .16 & $.20^{*}$ & - & & & \\
6. Tiempo & -.11 & .22 & .08 & .15 & .09 & - & & \\
7. Emociones positivas & $.31^{* *}$ & -.07 & .18 & -.01 & .03 & -.06 & - & \\
8. Emociones negativas & .08 & .19 & .09 & -.06 & -.17 & .00 & $-.24^{*}$ & - \\
\hline
\end{tabular}

Nota. ${ }^{*} p<.05,{ }^{* *} p<.01,{ }^{* * *} p<.001$; Sexo: 1 : Mujer, 2 : Hombre

\section{Tabla 4}

Predictores de la inmersión en condición de realidad virtual

\begin{tabular}{lcc}
\hline \multirow{2}{*}{ Variable } & \multicolumn{2}{c}{ Inmersión en condición de realidad virtual } \\
\cline { 2 - 3 } & \multicolumn{2}{c}{ Modelo 1} \\
\cline { 2 - 3 } Constante & 2.80 & {$[C 95 \%$} \\
Emociones & .34 & {$[.128-.562]$} \\
positivas & & \\
$R_{2}$ & & .095 \\
$F$ & & $9.95^{*}$ \\
\hline
\end{tabular}

Nota. $\mathrm{N}=97, \mathrm{IC}=$ Intervalo de confianza. ${ }^{*} p<.01$. Se presentan solo las relaciones significativas. 


\section{Discusión}

Se encontró evidencia para las cuatro hipótesis en estudio, a saber: la condición RV presenta mayor inmersión y mayor Frecuencia Cardiaca que la Condición No RV. Además, como parte de la evaluación de la activación emocional, se encontraron mayores emociones negativas después del juego en ambas condiciones. Esto último está relacionado con las características propias de un juego de terror (como el utilizado en este experimento) e incluso puede tener relación con el hecho de jugar solo (Lin, 2011; Ravaja et al, 2006).

Para respaldar la tercera hipótesis, se puede observar como evidencia el hecho de que los estados emocionales percibidos fueron predictores de la inmersión en ambos juegos, con la diferencia de que para la Condición No RV se obtuvieron mayores puntajes en la valoración de las emociones positivas y negativas, las cuales se asociaron a una mayor experiencia de inmersión, mientras que en la Condición RV solo las emociones positivas se relacionaron con la inmersión.

Los resultados encontrados resultan relevantes para entender el mecanismo de inmersión. Al ser un videojuego de terror era de esperarse la presencia de emociones negativas en ambas modalidades de juego. Ahora bien, resulta interesante que solo la emoción positiva fuera predictora de la inmersión, lo que de forma hipotética podría estar asociado con las implicaciones de experiencia misma de juego de la Realidad Virtual, ya que esta se caracteriza por la novedad donde la sensación de inmersión y presencia evocan un mayor disfrute, potenciando diversas emociones de este tipo, independientemente de que se trate de un juego de terror (de hecho en las pruebas piloto, los distintos participantes comentaban positivamente acerca de la "experiencia de susto" que les ofrecía el juego con RV).

El aumento de la frecuencia cardiaca, como muestra de la activación fisiológica, en la condición RV contribuye a apoyar esta hipótesis, ya que evidencia la evocación de mayores sensaciones que el juego No RV (Malinska, Zuzewicz, Bugajska, \& Grabowski, 2015). La condición de juego No RV, aunque puede ser que aporte disfrute, este 
podría ser menor, de forma tal que las experiencias de frustración o miedo podrían ser más evidentes que en el contexto RV donde la novedad de la sensación de inmersión puede adquirir un efecto predominante, opacando las otras.

$\mathrm{Al}$ respecto resulta relevante estudiar, en investigaciones futuras, las diferencias entre un juego de RV que sea de terror con uno que no lo sea. Asimismo, también es pertinente el estudio del papel que juegan las diferencias de personalidad en la experiencia de juego y dentro de estas; resulta relevante estimar el papel que juega la búsqueda de sensaciones como un factor importante para comprender las variaciones en la experiencia de inmersión y las emociones positivas y negativas.

De igual manera se hace necesario para entender el mecanismo de inmersión, continuar con el estudio de otros géneros de videojuego, de diferente demanda socio-cognitiva, de coordinación visomotora y atencional (por ejemplo, de aventura, resolución de retos o puzles, "shooters" o de disparar, etc.). Además, podría ser relevante estudiar diferencias según la modalidad de juego, ya sea esta una modalidad de juego individual o donde se juegue con otra persona, ya sea de forma offline o en línea (incluso considerando competitividad o trabajo colaborativo). Esto último podría estudiarse también, comparando la dinámica de interacción online en un mismo juego que ofrezca modalidad con y $\sin \mathrm{RV}$.

Finalmente se hace necesario aportar mayores evidencias de validez de la medida utilizada en este estudio, considerando igualmente diferentes tipos de videojuegos y poblaciones diferentes a la analizada. Cabe señalar que, para continuar estas investigaciones, este estudio ofrece, como parte de sus resultados, una versión validada de una escala de inmersión sobre los videojuegos (versión adaptada y traducida del Game Immersion Questionnaire de Cheng, Shet y Anneta, 2014), la cual resulta de mucha utilidad para estudios en esta temática. 


\section{Referencias}

Blanca, M. J., Alarcón, R., Arnau, J., Bono, R. y Bendayan, R. (2017). Non-normal data: Is ANOVA still a valid option?. Psicothema, 29(4), 552-557.

Cheng, M. T., Shet, H. C. y Anetta, L. A. (2014). Game Immersion Experience: its hierarchical structure and impact on game-based science learning. Journal of Computer assisted Learning, 31, 232-253.

Diemer, J., Lohkamp, N., Mühlberger, A., \& Zwanzger, P. (2016). Fear and physiological arousal during a virtual height challengeeffects in patients with acrophobia and healthy controls. Journal of Anxiety Disorders, 37, 30-39.

De Rooij, I. J. M., van de Port, I. G. L. y Meijer, J. G. (2016) Effect of Virtual Reality Training on Balance and Gait Ability in Patients With Stroke: Systematic Review and Meta-Analysis. Physical Therapy, 96 (12), 1905-1918.

Griffiths, M. D., Király, O., Pontes, H. M., \& Demetrovics, Z. (2015). An overview of problematic gaming. En E. Aboujaoude \& V. Starcevic (Eds.), Mental health in the digital age: Grave dangers, great promise (pp. 27-45). Oxford University Press. https://doi. org $/ 10.1093 / \mathrm{med} / 9780199380183.003 .0002$

Guerrero, B. y Valero, L. (2013). Efectos secundarios tras el uso de realidad virtual inmersiva en un videojuego. International Journal of Psychology and Psychological Therapy, 13(2), 163-178.

Gutierrez-Doña, B. (2003). Coping with stress at work. A longitudinal study on health outcomes and quality of life. Recuperado de http://www.diss.fu-berlin.de/2003/44/index.html

Hadley, W., Houck, C. D., Barker, D. H., Garcia, A. M., Spitalnick, J. S., Curtis, V., ... Brown, L. K. (2014). Eliciting affect via immersive virtual reality: a tool for adolescent risk reduction. Journal of Pediatric Psychology, 39(3), 358-368. 
Halbrook, Y. J., O’Donnell, A. T., \& Msetfi, R. M. (2019). When and How Video Games Can Be Good: A Review of the Positive Effects of Video Games on Well-Being. Perspectives on Psychological Science, 14(6), 1096-1104. https://doi. org/10.1177/1745691619863807

Hartanto, D., Kampmann, I. L., Morina, N., Emmelkamp, P. G. M., Neerincx, M. A., \& Brinkman, W.-P. (2014). Controlling social stress in virtual reality environments. Plos One, 9(3), e92804-e92804.

Hofer, M., Wirth, W., Kuehne, R., Schramm, H., \& Sacau, A. (2012). Structural Equation Modeling of Spatial Presence: The Influence of Cognitive Processes and Traits. Media Psychology, 15(4), 373-395. https://doi.org/10.1080/15213269.2012.723118

Ji, E., Lee, S. (2016). Effects of virtual reality training with modified constraint-induced movement therapy on upper extremity function in acute stage stroke: a preliminary study. The Journal of Physical Therapy Science, 28, 3168-3172.

Lee, K. M. (2004). Presence, Explained. Communication Theory, 14(1), 27-50.

Lin, S. (2011). Effect of Opponent Type on Moral Emotions and Responses to Video Game Play. Cyberpsychology, Behavior, and Social Networking, 14(11), 695-698.

Lopez-Fernandez, O., Williams, A.J., Griffiths, M.D. \& Kuss, D.J. (2019). Female gaming, gaming addiction, and the role of women within gaming culture: A narrative literature review. Frontiers in Psychiatry, 10, artículo 454, https://doi.org/10.3389/ fpsyt.2019.00454

Malinska, M., Zuzewicz, K., Bugajska, J., \& Grabowski, A. (2015). Heart rate variability (HRV) during virtual reality immersion. International Journal Of Occupational Safety And Ergonomics, 21(1), 47-54.

Notzon, S., Deppermann, S., Fallgatter, A., Diemer, J., Kroczek, A., Domschke, K., ... Ehlis, A.-C. (2015). Psychophysiological effects of an iTBS modulated virtual reality challenge including 
participants with spider phobia. Biological Psychology, 112, 66-76.

Nuyens FM, Kuss DJ, Lopez-Fernandez O, Griffiths MD (2018). The Empirical Analysis of Non-problematic Video Gaming and Cognitive Skills: A Systematic Review. Int J Ment Health Addict, 17, 389-414. https://doi.org/10.1007/s11469-018-9946-0

Ortiz de Gortari, A. B. y Griffiths, M. D. (2015). Game Transfer Phenomena and its associated factors: An exploratory empirical online survey study. Computers and Human Behavior, 51, 195-202.

Ravaja, N., Saari, T., Turpeinen, M., Laarni, J., Salminen, M. y Kivikangas, M. (2006). Spatial Presence and Emotions during Video Game Playing: Does It Matter with Whom You Play?. Presence, 15(4), 381-392.

Roettl J., \& Terlutter R. (2018). The same video game in 2D, 3D or virtual reality - How does technology impact game evaluation and brand placements? PLoS ONE 13(7): e0200724. https:// doi.org/10.1371/journal.pone.0200724

Roy, M. J., Costanzo, M. E., Jovanovic, T., Leaman, S., Taylor, P., Norrholm, S. D., y Rizzo, A. A. (2013). Heart rate response to fear conditioning and virtual reality in subthreshold PTSD. Studies in Health Technology And Informatics, 191, 115-119.

Ryan, R. M., Rigby, C. S. y Przybylski, A. (2006). The Motivational Pull of Videogames: A Self-Determination Theory Approach. Motivation and Emotion, 30, 347-363.

Schramm, H. (2012). The Role of Emotional Involvement and Trait Absorption in the Formation of Spatial Presence. Media Psychology, 15(1), 19-43. https://doi.org/10.1080/15213269.20 11.648536

Shafer, D. M., Carbonara, C. P. (2015). Examining Enjoyment of Casual Videogames. Games For Health Journal: Research, Development, and Clinical Applications, 4 (6), 1-8.

Vorderer, P. y Bryant, J. (2006). Playing Videogames. London: Routledge. 
Wirth, W., Hartmann, T., Böcking, S., Vorderer, P., Klimmt, C., Schramm, H., ... Jäncke, P. (2007). A Process Model of the Formation of Spatial Presence Experiences. Media Psychology, 9(3), 493-525. https://doi.org/10.1080/15213260701283079 Wirth, W., Hofer, M., \&

Yamato, T. P., Pompeu, J. E., Pompeu, S. M.A.A.A. y Hasset, L. (2016) Virtual Reality for Stroke Rehabilitation. Physical Therapy, 96(10), 1508-1513.

Recibido: 2019-11-03

Revisado: 2020-07-24

Aceptado: 2020-11-27 